\title{
SSSS
}

\section{How Do You Know You've Alternated?}

\section{Harry Collins}

Until I read Stolzenberg's (2004: 82) review, I had not realised I had really misread Bricmont and Sokal's argument: I thought they were just 'nitpicking'. I'm still not so sure, but I'll leave that to readers of the book. I'll concentrate on the more substantive question of 'alternation' discussed in his last paragraph. Stolzenberg (2004: 86) points out that he has been told that good sociologists acquire the skill of alternating between world views during their training but that he is suspicious:

Not only do they make it seem too easy 'to take on the ways of being in the world that are characteristic of the groups they study', but they also fail to explain how they know when they have gotten it right. Nor do they say whether, when they 'return home', they have any better luck communicating their findings than did Square, upon his return to Flatland from Spaceland.

These are very reasonable suspicions and in the spirit of The One Culture?, which tries to move things forward, I'll address them in a positive way.

Let me say first that these are problems that continually engage social scientists and they are far from settled among our community. Exactly the same suspicions could very reasonably be expressed by a practising social scientist and we talk about these things all the time even in our own regular Cardiff seminar groups with our own colleagues and graduate students. We think we have a pretty good answer to the first problem - how do we know we've achieved a degree of 'native competence' - but all we can do is 'make a stab' at communicating with the folks back home.

As to the first problem, we have recently decided that it is useful to distinguish between two kinds of expertise: 'contributory expertise' and 'interactional expertise' (Collins \& Evans, 2002). In the case of a science, such as gravitational wave physics, contributory expertise is what you need in order to be, say, offered a job as a gravitational wave physicist. Interactional expertise is what you need to have an interesting argument with a gravitational wave physicist about gravitational wave physics with a tiny chance that you might occasionally score a point. In other words, if you have interactional expertise, you understand the principles of the science 
or technology pretty well, but not well enough to make a regular substantive contribution to it. Nevertheless, interactional expertise takes quite a long time to learn and you can fail to learn it (as Collins failed to learn the interactional expertise of the theory of amorphous semiconductors even after trying very hard for a long time). Interactional expertise is also to be distinguished from parrot-like learning facts about a subject. Interactional expertise enables you to retrieve relevant facts and ideas so as to maintain a lively conversation. Even the most 'intelligent' computer, stocked with the most exhaustive set of facts about a subject, cannot do that (see for example, Collins, 1990).

We have some arguments that show, we believe, that interactional expertise in a subject is good enough to do the sociology of the subject in the absence of contributory expertise. To establish this, we look at sociologists who do have contributory expertise in the subjects they analyse sociologically (for example, Collins in parapsychology; Pickering in highenergy physics), and note that their sociology is no better in virtue of that higher level of expertise. We note also, however, that if you fail to master interactional expertise (as Collins in the case of the theory of amorphous semiconductors), you cannot do the sociology (of knowledge) of the subject.

Stolzenberg's question becomes, then: how do you know you have acquired interactional expertise in a subject? The answer is in the ways you can interact. As a sociologist of science you essay research on a new specialism and you initially understand neither the banter nor the technical terms. After a painful period, if you are lucky you begin to pick up on the inferences in others' conversations and eventually you begin to be able to join in. One day a respondent might say in response to one of your technical queries 'I had not thought about that', and pause before giving you an answer. When this stage is reached respondents will start to be happy to talk to you about physics and even respond generously and with consideration to your critical comments. Eventually people will become interested in what you know, not as a scientist in your own right, but as a person who is able to convey the scientific thoughts and activities of others. If you've just come from visiting scientist $\mathrm{X}$ you may be able to tell scientist $\mathrm{Y}$ something of the science that $\mathrm{X}$ is doing. (I could never have done this in the case of the theory of amorphous semiconductors because I never learned to describe what people were thinking in a scientifically coherent way.) What were once 'interviews' then become 'conversations' that can be interesting and occasionally even useful to both parties. What also happens in a conversation is that by occasionally anticipating a point your partner is about to make you can speed things along. You might also verbally fill in some gaps that might otherwise be forgotten. You can recognize jokes, irony and when you are having your leg pulled (though, in the nature of things, interactional competence does not allow you to recognize lies). When you get good at it you can even take the devil's advocate position in respect of some scientific controversy and maintain it well enough to make your conversational partner think hard. 
Generally, you know when you are accomplishing these things by the way the conversation goes but, if there can be something that amounts to 'proof' in such a case it lies in the reaction of your respondents when you pass them draft analyses to read. If they contain scientific faux pas you will soon know. That interactional expertise has been acquired may be hard to prove to an outsider but indicators of acquisition to the acquirer are quite robust.

Now to the more difficult problem: how can we be sure that we are succeeding in putting across our new-found knowledge to the folks back home - the readers? This is not just a problem for us, it is a problem for all authors. The shock of what readers read into what you write must have been experienced since the early days of writing. Certainly, almost everything I write, and that includes the straightforward pieces, seems open to astonishing misinterpretation by at least a few people. And since Roland Barthes' 'death of the author' we've all been academically aware that this is likely to be the case as well as viscerally aware. So nothing I can say here is going to carry much assurance. But, as Stolzenberg implies, we can have even less confidence in our attempts to convey our internal states to our readers than regular authors, since we know how hard it is to acquire interactional expertise and we also know about socialization, tacit knowledge and all the rest of it; we know that the written word cannot substitute for years of face-to-face interaction.

The 'stab at it' that I promised earlier is simply this: we use our respondents' own words in an attempt to convey social life; this amounts to a groping attempt to borrow the techniques of the novelist. Somehow novelists sometimes seems to transport you to another world, and though we are not trained as writers we hope that the liberal use of quotations selected because they seem to us to convey the social nuance that we were experiencing at the time - will go some way to accomplishing the same. But the paradox remains, just as Stolzenberg says.

Finally, let me say that Stolzenberg is a little unfair on his scientist colleagues when he says they have made no progress in learning to alternate between world views. First, it should be remembered that social scientists start with a tremendous advantage in this respect; pretty well all of us who make science their substantive subject of study have been inducted into the sciences at some stage of their careers. I am one of the least qualified of the social scientists, but even I learned the thrill of doing an experiment or solving a mathematical problem when I was at (high) school. I learned what it was to experience the force of an external reality and the force of suddenly coming to understand it in a beautiful, systematic and universalistic way; I still do experiments, such as building a model interferometer, to keep myself in touch with these forces. And building a model interferometer, or whatever, is much easier than learning to estrange yourself from taken-for-granted reality.

Among the more bizarre of the criticisms we encountered at the height of the science wars was that we did not know what an experiment/theory was; there are very few sociologists of science who do not know it (though 
the situation becomes less clear when we move to cultural studies of science), and it is nice that Steven Weinberg acknowledges this in the book. Of course, this kind of general understanding of 'what makes science tick' is only a foundation for the building of interactional expertise in a specific scientific field.

But having been well and truly socialized into science, I then spent years as a social scientist learning to un-understand the force of experiment and theory. Scientist colleagues have not had the advantage of these two lots of socialization - in the main they have had only one. The mistake that a lot of scientists make is thinking that the skill of un-understanding experiment and theory is non-existent - that all there is to know about it can be gained by reading a couple of articles or a book. This is not so. Nevertheless, it seems to me that at least some scientists, and without mentioning names let me say that some of these are to be found among the contributors to The One Culture?, have in fact made good progress in learning the alternation trick. One of the great joys of editing the book was discovering that this generation of scholars was really beginning to extend their understanding (and ability to alternate), across the 'two cultures' divide, and that things were going both ways.

\section{References}

Collins, H.M. (1990) Artificial Experts: Social Knowledge and Intelligent Machines (Cambridge, MA: MIT Press).

Collins, H.M. \& Robert Evans (2002) 'The Third Wave of Science Studies: Studies of Expertise and Experience', Social Studies of Science 32(2): 235-96.

Stolzenberg, Gabriel (2004) 'Kindler, Gentler Science Wars', Social Studies of Science 34(1): $77-89$.

Harry Collins is Distinguished Research Professor of Sociology and Director of the Centre for the Study of Knowledge, Expertise and Science (KES) at Cardiff University.

Address: KES, Cardiff School of Social Science, Cardiff University, The Glamorgan Building, King Edward VII Avenue, Cardiff CF10 3WT, UK; fax: +44 2920 874175; email: CollinsHM@cardiff.ac.uk 